\title{
Incidence of adjacent segment degeneration in cervical disc arthroplasty versus anterior cervical decompression and fusion meta-analysis of prospective studies
}

\author{
Jiaquan Luo • Ming Gong • Sheng Huang • \\ Ting Yu $\cdot$ Xuenong Zou
}

Received: 13 August 2014/Published online: 26 November 2014

(C) The Author(s) 2014. This article is published with open access at Springerlink.com

\begin{abstract}
Purpose To evaluate the incidence of adjacent segment disease (ASD) requiring surgical intervention between anterior cervical decompression and fusion (ACDF) and total disc replacement (TDR).

Background The concern for ASD has led to the development of motion-preserving technologies such as TDR. However, whether replacement arthroplasty in the spine achieves its primary patient-centered objective of lowering the frequency of adjacent segment degeneration is not verified yet.

Methods A comprehensive literature search was performed using PubMed, Cochrane Central Register of Controlled Trials and Embase. These databases were thoroughly searched for prospective randomized studies comparing ACDF and TDR. Eight studies met the inclusion criteria for a meta-analysis and were used to report an overall rate of ASD for both ACDF and TDR.

Results Pooling data from 8 prospective studies, the overall sample size at baseline was 1,726 patients (889 in the TDR group and 837 in the ACDF group). The ACDF group had significantly more ASDs compared with the TDR group at 24 months postoperatively [odds ratios (OR), 1.31; $95 \%$ confidence interval (CI), 1.04-1.64; $p=0.02]$. The TDR group had significantly fewer adjacent segment reoperations compared with the ACDF group at
\end{abstract}

J. Luo $\cdot$ M. Gong $\cdot$ S. Huang $\cdot$ T. Yu $\cdot$ X. Zou $(\bowtie)$ Department of Spine Surgery/Orthopaedic Research Institute, The First Affiliated Hospital of Sun Yat-sen University, Guangzhou 510080, People's Republic of China e-mail: zxnong@hotmail.com

J. Luo

e-mail: luojiaquan666@163.com
24 months postoperatively (OR, 0.49; $95 \%$ CI, 0.25-0.96; $p=0.04)$.

Conclusions For patients with one-level cervical degenerative disc disease (CDDD), total disc replacement was found to have significantly fewer ASDs and reoperations compared with the ACDF. Cervical replacement arthroplasty may be superior to ACDF in ASD. Therefore, cervical arthroplasty is a safe and effective surgical procedure for treating CDDD. We suggest adopting TDR on a large scale.

Keywords TDR $\cdot A C D F \cdot A S D$

\section{Introduction}

Symptomatic myelopathy and/or radiculopathy are common indications for surgical intervention in the cervical spine. Anterior cervical decompression and fusion (ACDF) has been widely performed and considered as the standard surgical treatment for cervical degenerative disc disease (CDDD). Fusion of the cervical spine has biomechanical consequences. Loss of motion at the operated spinal unit increases the load sustained by the adjacent units [1]. Previous study has demonstrated that anterior cervical fusion is associated with the adjacent segment degeneration (ASD) [2]. However, the cause for ASD remains widely controversial. Some scholars believe that incidence represents a natural progression of cervical disc disease, whereas others suggest altered biomechanics at levels adjacent to a fusion accelerate this process [3, 4].

Cervical disc prostheses are designed to preserve motion patterns and disc height, to avoid the limitations of fusion, and to maintain normal segmental lordosis after surgery. Previous studies have demonstrated that artificial disc 
arthroplasty offers the theoretical advantage of preservation of motion at the operative level with consequent stress reduction at adjacent levels [5, 6]. However, few clinical studies have specifically aimed to evaluate adjacent segment degeneration after cervical disc arthroplasty.

Whether cervical replacement arthroplasty will achieve its original patient-centered goals with improved outcomes and less adjacent segment degeneration remains an unresolved issue. To further clarify this debate, we perform a meta-analysis of the current available evidence comparing the reported incidence of ASD requiring surgical intervention between ACDF and TDA. This study also aims to emphasize the importance of reporting ASD as an outcome in future prospective studies.

\section{Materials and methods}

\section{Search strategy}

We searched for randomized controlled trials (RCTs) published between January 1960 and June 2014 that compared cervical arthroplasty with ACDF in patients with cervical radiculopathy or myelopathy. The databases included PubMed, Cochrane Central Register of Controlled Trials, and Embase with no language restriction. In addition, we also performed handsearching of information in the Orthopedics China Biological Medicine Database. The following search terms were used: "cervical disc replacement", "disc replacement", "cervical artificial disc replacement", "cervical disc arthroplasty", and RCT.

\section{Inclusion criteria}

The inclusion criteria were: (1) randomized, controlled study of degenerative disc disease of the cervical spine involving single segment or double segments using CDA with anterior cervical discectomy and fusion (ACDF) as controls; (2) a minimum of 2-year follow-up using imaging and clinical analyses; (3) definite diagnostic evidences for "adjacent segment degeneration" and "adjacent segment disease".

\section{Exclusion criteria}

Exclusion criteria were: (1) case reports; (2) reviews; (3) patients with cervical spine disease involving more than three segments.

Study selection

Two of the authors (J.-Q.L. and S.H.) independently screened the article titles and abstracts based on the eligibility criteria. Intensive reading of the full text was performed when the studies met the inclusion criteria. Disagreements were resolved by discussion to reach a consensus.

\section{Data extraction}

Relevant data were extracted independently by two authors (J.-Q.L. and M.G.). The data included the general characteristics of each study and the outcomes measured. General characteristics included study design, first author, year of publication, sample size, interventions and various types of artificial total disc replacements (TDRs). The outcomes measured included: the rate of postoperative development of adjacent segment degenerative or diseases and the rate of adjacent segment surgery. Discrepancies were resolved through discussion.

Quality assessment

According to the Cochrane Handbook for Systematic Reviews of Interventions, version 5.0, the quality of the studies was independently evaluated by two authors (J.Q.L. and T.-Y.). The following domains were assessed: randomization, blinding (of patients, surgeons, and assessors), allocation concealment, and follow-up coverage. Each domain of quality assessment was classified as adequate (A), unclear (B), or inadequate (C).

\section{Data analysis}

We performed all meta-analyses with the Review Manager software (RevMan Version 5.1; The Nordic Cochrane Center, The Cochrane Collaboration, Copenhagen, Denmark). Only dichotomous outcomes were mentioned in our study, so the OR or risk ratios and $95 \%$ confidence intervals were calculated for outcomes. A probability of $p<0.05$ was considered to be statistically significant. Assessment for statistical heterogeneity was calculated using the Chi-square and $I$-square tests. $I^{2}$ ranges from 0 to $100 \%$, with $0 \%$ indicating the absence of any heterogeneity. Although absolute numbers for $I^{2}$ are not available, values $<50 \%$ are considered low heterogeneity. When $I^{2}$ is $<50 \%$, low heterogeneity is assumed, and the effect is thought to be due to change. Conversely, when $I^{2>} 50 \%$, heterogeneity is thought to exist and the effect is random.

\section{Results}

The process of identifying relevant studies is summarized in Fig. 1. From the selected databases, 175 references were obtained. By screening the titles and abstracts, 107 references were excluded due to the duplicates and irrelevance to this topic. The remaining 68 reports underwent a detailed and 


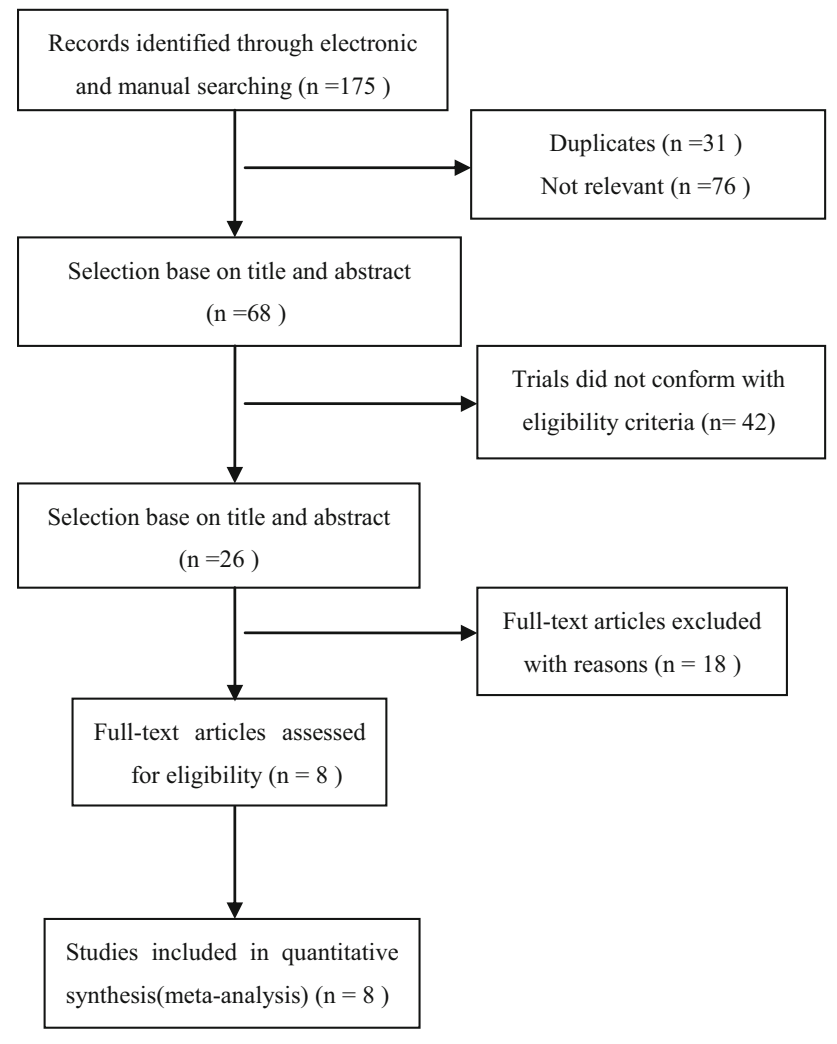

Fig. 1 The flow chart shows the article selection process we performed

comprehensive evaluation. Finally, 8 RCTs were included in this meta-analysis [7-14]. The main characteristics of included studies are summarized in Table 1.

Quality assessment

The results of the quality assessment are presented in Table 2. Of the eight studies, eight stated the exact randomization methods used [7-14]. Two studies blinded both the patients and the assessors [12, 14]. None of the studies documented concealment of randomization. Demographic data at baseline were similar in the two treatment groups. Descriptions of patient drop-outs and withdrawals appeared in all eight reports. Hence, the methodological quality of the eight studies included was level B.

Surgical parameters

\section{Adjacent segment disease}

Adjacent segment disease was provided in 8 studies, and all these studies with a total of 1,726 patients (889 in the TDR group and 837 in the ACDF group) were analyzed. The ACDF group had significantly more adjacent segment diseases compared with the TDR group at 24 months postoperatively (OR, 1.31; $95 \% \mathrm{CI}, 1.04-1.64 ; p=0.02$ ) (Fig. 2).

\section{Adjacent segment reoperations}

Adjacent segment reoperations were provided in 4 studies with a total of 1,066 patients (536 in the TDR group and 530 in the ACDF group) analyzed. The TDR group had significantly fewer adjacent segment reoperations compared with the ACDF group at 24 months postoperatively (OR, 0.49; $95 \%$ CI, 0.25-0.96; $p=0.04$ ) (Fig. 3).

\section{Discussion}

In this meta-analysis, we identified 8 randomized clinical trials with up to minimum 24 months of follow-up

Table 1 Characteristics of studies included in the meta-analysis of cervical arthroplasty compared to anterior cervical discectomy and fusion for treating one-level cervical disc disease

\begin{tabular}{|c|c|c|c|c|c|c|c|c|c|}
\hline \multirow[t]{2}{*}{ Studies } & \multirow[t]{2}{*}{ Design } & \multirow[t]{2}{*}{ Country } & \multicolumn{2}{|c|}{ Sample size } & \multicolumn{2}{|c|}{ Mean age (years) } & \multicolumn{2}{|c|}{ Gender (M/F) } & \multirow[t]{2}{*}{ Follow-up (months) } \\
\hline & & & TDR & $\mathrm{ACDF}$ & TDR & $\mathrm{ACDF}$ & TDR & $\mathrm{ACDF}$ & \\
\hline Porchet [7] & RCT 4 centers & Switzerland & 27 & 28 & $44 \pm 8.9$ & $43 \pm 6.9$ & $17 / 10$ & $12 / 16$ & 24 \\
\hline Murrey [8] & RCT 13 centers & USA & 103 & 106 & $42.1 \pm 8.4$ & $43.5 \pm 7.1$ & $46 / 57$ & $49 / 57$ & 24 \\
\hline Nabhan [9] & RCT 1 centers & Germany & 20 & 21 & 44 & & $23 / 18$ & & 36 \\
\hline Garrido [10] & RCT 1 centers & USA & 21 & 26 & 40 & 43.3 & $13 / 8$ & $26 / 14$ & 48 \\
\hline Burkus [11] & RCT 32 centers & USA & 276 & 265 & 43.3 & 43.9 & $128 / 148$ & $122 / 143$ & 60 \\
\hline Sasso [12] & RCT 31 centers & USA & 242 & 221 & $44.4(25-78)$ & $44.7(27-68)$ & $110 / 132$ & $113 / 108$ & 48 \\
\hline Coric [13] & RCT 21 centers & USA & 136 & 133 & $43.7 \pm 7.76$ & $43.9 \pm 7.39$ & $51 / 85$ & $59 / 74$ & 24 \\
\hline Jawahar [14] & RCT 1 centers & USA & 59 & 34 & - & - & $21 / 38$ & $16 / 18$ & 24 \\
\hline
\end{tabular}

Mean age was described as mean $\pm \mathrm{SD}$ or mean (range)

$A C D F$ anterior cervical discectomy and fusion, $R C T$ randomized control trial, $S D$ standard deviation, $T D R$ total disc replacement, $M$ male, $F$ female 
Table 2 Methodological quality of studies included in the meta-analysis of cervical arthroplasty compared to ACDF for treating one-level CDDD

\begin{tabular}{|c|c|c|c|c|c|c|c|}
\hline \multirow[t]{2}{*}{ Years } & \multicolumn{3}{|l|}{ Baseline } & \multirow[t]{2}{*}{ Randomization } & \multirow[t]{2}{*}{ Allocation concealment } & \multirow[t]{2}{*}{ Blinding } & \multirow[t]{2}{*}{ Quality level } \\
\hline & Size & Age & Sex & & & & \\
\hline Porchet [7] & Comparable & Comparable & Comparable & Adequate & Unclear & Unclear & $\mathrm{B}$ \\
\hline Murrey [8] & Comparable & Comparable & Comparable & Adequate & Unclear & Unclear & B \\
\hline Nabhan [9] & Comparable & Comparable & Comparable & Adequate & Unclear & Unclear & B \\
\hline Garrido [10] & Comparable & Comparable & Comparable & Adequate & Unclear & Unclear & B \\
\hline Burkus [11] & Comparable & Comparable & Comparable & Adequate & Unclear & Unclear & B \\
\hline Sasso [12] & Comparable & Comparable & Comparable & Adequate & Unclear & Double & B \\
\hline Coric [13] & Comparable & Comparable & Comparable & Inadequate & Unclear & Single & B \\
\hline Jawahar [14] & Comparable & Comparable & Comparable & Adequate & Unclear & Double & $\mathrm{B}$ \\
\hline
\end{tabular}

Comparable: the variables were comparable among all studies

Each domain of quality assessment was classified as: adequate (A), unclear (B), or inadequate (C)

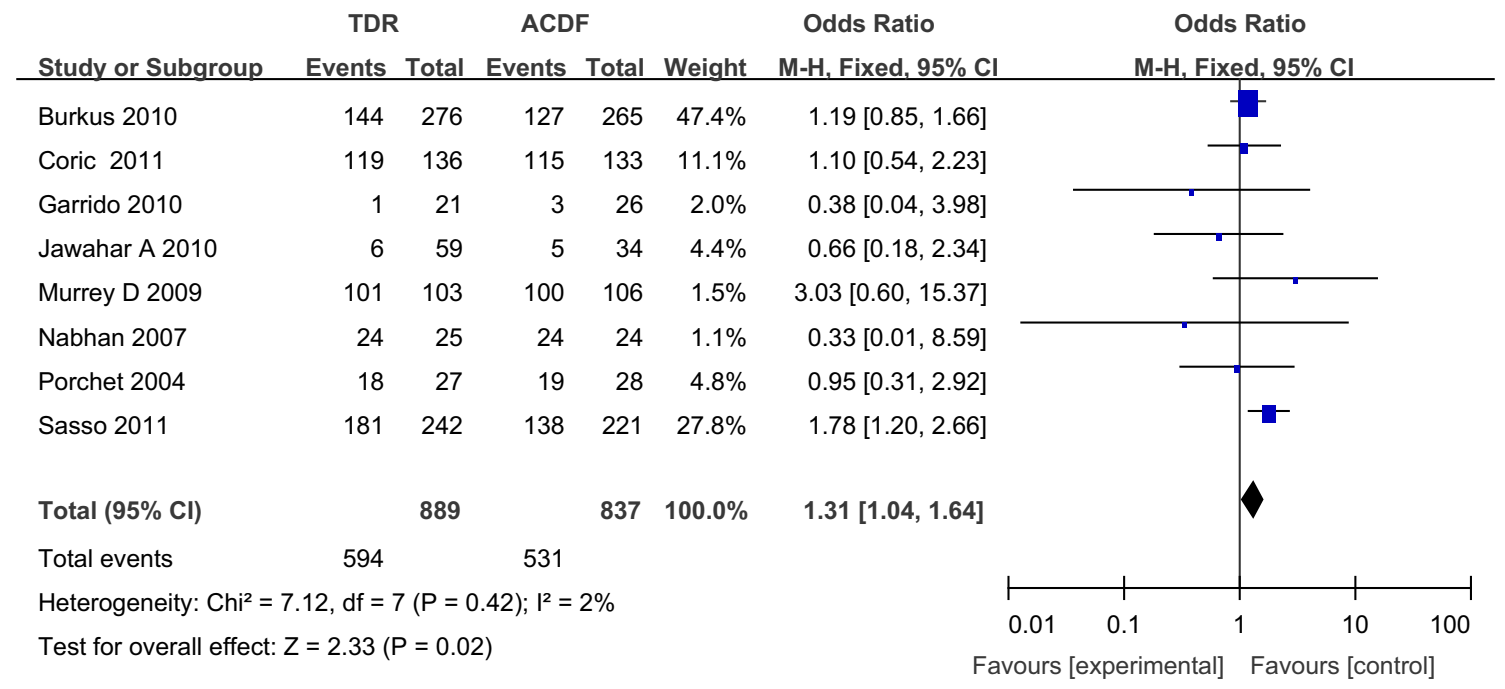

Fig. 2 Forest plot of adjacent segment disease for the TDR and ACDF groups at 24 months postoperatively. $A C D F$ anterior cervical discectomy and fusion, $T D R$ total disc replacement, $C I$ confidence interval, $M-H$ Mantel-Haenszel, $S D$ standard deviation

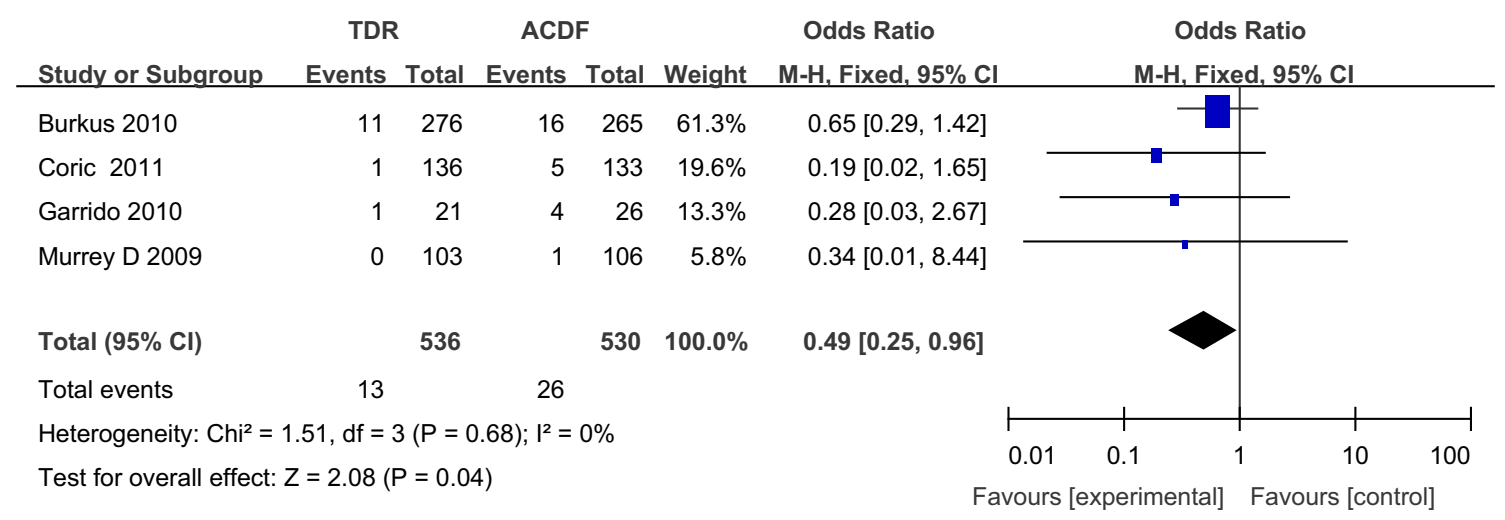

Fig. 3 Forest plot of adjacent segment reoperations for the TDR and ACDF groups at 24 months postoperatively. ACDF anterior cervical discectomy and fusion, $T D R$ total disc replacement, $C I$ confidence interval, $M-H$ Mantel-Haenszel, $S D$ standard deviation 
assessing the effects of TDR for patients with one-level CDDD refractory to nonoperative treatment. We found that the TDR group had significantly fewer adjacent segment diseases and reoperations compared with the ACDF.

A few meta-analyses have been published recently comparing ACDF versus TDR. Results of our meta-analyses showed that TDR group had significantly fewer adjacent segment reoperations compared with the ACDF group at 24 months postoperatively. Gao et al. [15] reported that arthroplasty was associated with fewer secondary surgical procedures. However, the indications for these secondary procedures were unclear. From the 2-year follow-up data, Phillips et al. [16] reported an equivalent rate of secondary procedures for ASD versus TDR (5.4 versus $5.2 \%$ ). However, the study does not specifically report the number of reoperations for ASD alone.

With regard to ASD, our meta-analysis showed that the ACDF group had significantly more ASD compared with the TDR group at 24 months postoperatively.

A recent meta-analysis by Jiang et al. [17] also found a lower rate of ASD for TDR versus ACDF. However, this analysis included radiographical assessments of ASD that do not correlate with reoperation rate. In addition, the analysis was heavily influenced by the 2-year follow-up data from Mummaneni et al. [18]. The 5-year data from this same author, however, showed equivalent ASD rates for ACDF versus TDR [19]. Verma et al. concluded there is no difference in the rate of ASD for ACDF versus TDA. They report an overall lower rate of follow-up for patients with ACDF than for those with TDR [20]. Similarly, Yang et al. reported no difference in the incidence of ASD (radiographical features and reoperation rate) comparing ACDF versus TDR in a meta-analysis. Although the conclusion of the authors was similar to that of this study, there were methodological differences worth nothing [21]. In addition, Yang et al. [21] included only 140 patients in the meta-analysis, whereas this study included more than 1,500 patients at baseline and 1,100 patients at the final followup. Lastly, the meta-analysis by Yang et al. [21] was update to 2011, but their selection of studies was notably different than that of this study.

There are many and complicated reasons for developing ASD after ACDF and arthroplasty, such as the increased adjacent vertebral sagittal activity [22], the fusion segment number [23], the segment locations [23], segmental kyphosis operation [24], and the influence of each factor on the other. Increased stress of fused adjacent segments is the reason of causing ASD [25]. A biomechanical and kinematic study suggested that preservation of motion at the operated level might help to lessen the incidence of adjacent-level disc degeneration [26]. TDR is developed to restore physiologic biomechanics and to reduce the adjacent-level forces, thereby reducing the potential for accelerated adjacent-level disc degeneration [27]. However, whether cervical replacement arthroplasty will achieve its original patient-centered goals with improved outcomes and less adjacent segment degeneration remains unclear.

In our meta-analysis, eight published RCTs on cervical TDR versus fusion were analyzed. Seven studies had good methodological qualities (Jadad scores $\geq 4$ ); one study only gained three scores which implied a higher risk of bias. The most prevalent methodological shortcoming appeared to be insufficiency regarding the outcome assessor blinding to intervention. The low number of included studies limited our assessment of potential publication bias by the funnel plot and unpublished researches with negative results cannot be identified. Therefore, publication bias may exist, which could result in the overestimation of the effectiveness of interventions.

We believe that our result of meta-analysis is affected by several reasons. First, the number of articles may be insufficient and we included only eight studies in the evaluation, what might have led to an insufficient significant effectiveness. Second, the low number of included studies limited our assessment of a potential publication bias which cannot be excluded due to unpublished negative research results. Therefore, publication bias may exist, which might have resulted in the overestimation of the intervention effectiveness. Third, the properties of the different prostheses, the various indications for surgery, and the surgical technologies used at different treatment centers. Due to these limitations, the combined results of this meta-analysis should be cautiously accepted, and highquality RCTs with long-term follow-up and large sample size are needed.

In summary, our meta-analysis indicated, for the treatment of CDDD, that cervical disc arthroplasty had significantly fewer adjacent segment diseases and reoperations compared with the ACDF. TDR may be superior to ACDF in ASD.

\section{Conclusion}

For patients with one-level CDDD, TDR was found to have significant fewer adjacent segment diseases and reoperations compared with the ACDF. TDR may be superior to ACDF in ASD. Therefore, TDR is a safe and effective surgical procedure for treating CDDD. We suggest adopting TDR on a large scale.

Acknowledgments The present study was financially supported by National Program on Key Basic Research Project (973 Program; Grant No. 2012CB619105). 
Conflict of interest The authors declare that they have no conflicts of interest concerning this article.

Open Access This article is distributed under the terms of the Creative Commons Attribution License which permits any use, distribution, and reproduction in any medium, provided the original author(s) and the source are credited.

\section{References}

1. Matsunaga S, Kabayama S, Yamamoto T, Yone K, Sakou T et al (1999) Strain on intervertebral discs after anterior cervical decompression and fusion. Spine 24:670-675

2. Hilibrand AS, Carlson GD, Palumbo M, Jones PK, Bohlman HH et al (1999) Radiculopathy and myelopathy at segments adjacent to the site of a previous anterior cervical arthrodesis. J Bone Joint Surg Am 81:519-528

3. Lund T, Oxland TR (2011) Adjacent level disk disease-is it really a fusion disease? Orthop Clin North Am 42(4):529-541

4. Hilibrand AS, Robbins M (2004) Adjacent segment degeneration and adjacent segment disease: the consequences of spinal fusion? Spine J 4(6 suppl):190S-194S

5. DiAngelo DJ, Roberston JT, Metcalf NH et al (2003) Biomechanical testing of an artificial cervical joint and an anterior cervical plate. J Spinal Disord Tech 16:314-323

6. Wigfield C, Gill S, Nelson R, et al (2002) Influence of an artificial cervical joint compared with fusion on adjacent-level motion in the treatment of degenerative cervical disc disease. J Neurosurg 96(1suppl):17-21

7. Porchet F, Metcalf NH (2004) Clinical outcomes with the Prestige II cervical disc: preliminary results from a prospective randomized clinical trial. Neurosurg Focus 17:E6

8. Murrey D, Janssen M, Delamarter R et al (2009) Results of the prospective, randomized, controlled multicenter Food and Drug Administration investigational device exemption study of the ProDisc-C total disc replacement versus anterior discectomy and fusion for the treatment of 1-level symptomatic cervical disc disease. Spine J 9:275-286

9. Nabhan A, Steudel WI, Nabhan A et al (2007) Segmental kinematics and adjacent level degeneration following disc replacement versus fusion: RCT with three years of follow-up. J Long Term Eff Med Implants 17(3):229-236

10. Garrido BJ, Taha TA, Sasso RC (2010) Clinical outcomes of Bryan cervical disc arthroplasty a prospective, randomized, controlled, single site trial with 48-month follow-up. J Spinal Disord Tech 23(6):367-371

11. Burkus JK, Haid RW, Traynelis VC et al (2010) Long-term clinical and radiographic outcomes of cervical disc replacement with the Prestige disc: results from a prospective randomized controlled clinical trial. J Neurosurg Spine 13(3):308-318

12. Sasso RC, Anderson PA, Riew KD et al (2011) Results of cervical arthroplasty compared with anterior discectomy and fusion: four-year clinical outcomes in a prospective, randomized controlled trial. J Bone Joint Surg Am 93:1684-1692

13. Coric D, Nunley PD, Guyer RD et al (2011) Prospective, randomized, multicenter study of cervical arthroplasty: 269 patients from the KineflexlC artificial disc investigational device exemption study with a minimum 2-year follow-up: clinical article. J Neurosurg Spine 15:348-358

14. Jawahar A, Cavanaugh DA, Kerr EJ 3rd, Birdsong EM, Nunley PD et al (2010) Total disc arthroplasty does not affect the incidence of adjacent segment degeneration in cervical spine: results of 93 patients in three prospective randomized clinical trials. Spine 10:1043-1048

15. Gao Y, Liu M, Li T et al (2013) A meta-analysis comparing the results of cervical disc arthroplasty with anterior cervical discectomy and fusion (ACDF) for the treatment of symptomatic cervical disc disease. J Bone Joint Surg Am 95:555-561

16. Phillips FM, Lee JY, Geisler FH et al (2013) A prospective, randomized, controlled clinical investigation comparing $\operatorname{PCM}(\mathrm{r})$ cervical disc arthroplasty to anterior cervical discectomy and fusion: 2 year results from the us IDE clinical trial. Spine 38(15):E907-E918

17. Jiang H, Zhu Z, Qiu Y et al (2012) Cervical disc arthroplasty versus fusion for single-level symptomatic cervical disc disease: a meta-analysis of randomized controlled trials. Arch Orthop Trauma Surg 132:141-151

18. Mummaneni PV, Burkus JK, Haid RW et al (2007) Clinical and radiographic analysis of cervical disc arthroplasty compared with allograft fusion: a randomized controlled clinical trial. J Neurosurg Spine 6:198-209

19. Robertson JT, Papadopoulos SM, Traynelis VC (2005) Assessment of adjacent-segment disease in patients treated with cervical fusion or arthroplasty: a prospective 2-year study. J Neurosurg Spine 3:417-423

20. Verma K, Gandhi SD, Maltenfort M et al (2013) Rate of adjacent segment disease in cervical disc arthroplasty versus single-level fusion: meta-analysis of prospective studies. Spine 38(26):2253-2257

21. Yang B, Li H, Zhang $\mathrm{T}$ et al (2012) The incidence of adjacent segment degeneration after cervical disc arthroplasty (CDA): a meta analysis of randomized controlled trials. PLoS One 7(4):e35032

22. Hilibrand AS, Carlson GD, Palumbo M, Jones PK, Bohlman HH et al (1999) Radiculopathy and myelopathy at segments adjacent to the site of a previous anterior cervical arthrodesis. J Bone Joint Surg Am 81:519-528

23. Takeshima T, Omokawa S, Takaoka T, Araki M, Ueda Y et al (2002) Sagittal alignment of cervical flexion and extension: lateral radiographic analysis. Spine 27:348-355

24. Goffin J, Geusens E, Vantomme N (2004) Long term follow-up after interbody fusion of the cervical spine. Spinal Disord Tech 17:79-85

25. Eck JC, Humphreys SC, Lim TH et al (2002) Biomechanical study on the effect of cervical spine fusion on adjacent-level intradiscal pressure and segmental motion. Spine 27(22):2431-2434

26. Dmitriev AE, Cunningham BW, $\mathrm{Hu} \mathrm{N}$ et al (2005) Adjacent level intradiscal pressure and segmental kinematics following a cervical total disc arthroplasty: an in vitro human cadaveric model. Spine 30:1165-1172

27. Duggal N, Pickett GE, Mitsis DK et al (2004) Early clinical and biomechanical results following cervical arthroplasty. Neurosurg Focus 17:E9 